\title{
Applying the Search Strategies Approach to Practice: The Feldenkrais Method
}

\author{
CHARLEY W. LAFE ${ }^{1}$ | MATHEUS M. PACHECO2,3 | \\ ${ }_{1}^{1}$ Rehab Neural Engineering Lab, School of Medicine, University of Pittsburgh, USA. \\ 2 Motor Behavior Laboratory (LACOM), School of Physical Education and Sport, University of São Paulo, São Paulo, SP, Brazil \\ 3 Motor Development Study Group (GEDEM), Federal University of Rondônia, Porto Velho, RO, Brazil.
}

Correspondence to: Matheus M. Pacheco, Av. Prof. Mello Moraes, 65 - Cidade Universitária, CEP: 05508-030 - São Paulo - SP, BRAZIL.

email: matheus.lacom@gmail.com

https://doi.org/10.20338/bjmb.v13i5.147

HIGHLIGHTS

- The search strategies approach (SSA) has shown potential in controlled experiments.

- The Feldenkrais Method (FM) is a nontraditional rehabilitation method.

- The SSA can be linked to FM through the view of skill acquisition as search.

\section{ABBREVIATIONS}

FM Feldenkrais Method

SSA search strategies approach

PUBLICATION DATA

Received 31102019

Accepted 30112019

Published 01122019
BACKGROUND: The search strategies approach (SSA) to skill acquisition has its roots in the dynamical systems approach and has demonstrated a number of theoretical advances to the area. We argue that its advances can and are already linked to practical applications when we consider the method of Moshe Feldenkrais. The Feldenkrais Method (FM) considers skill acquisition (from rehabilitation to daily life activities improvement) in terms of exploration of new possibilities to achieve efficiency in movement. In this paper, we discuss how the SSA encompasses Feldenkrais method (FM) illustrating its applicability. Furthermore, we point to new possibilities in both FM and SSA if these are to be formally linked.

\section{INTRODUCTION}

The search strategies approach (SSA) to motor learning ${ }^{1,2}$ integrates different lines of research of the Dynamical Systems Approach to motor behavior emphasizing the individualistic nature of skill acquisition through formal evaluation of task and environmental constraints. While in its early development, SSA promises to have large influence beyond its theoretical pursuits. At face level, its practical application may not be so apparent. In this paper we argue that there exists already a therapeutic method - called 'the Feldenkrais Method' (FM) - that resembles and embodies the SSA.

FM provides a unique way of improving quality of movement. Its application ranges from rehabbing an injury, a movement related disease, or simply improving activities of daily living ${ }^{3-5}$. Founded on a singular perspective about how movements are developed and learned, it has, unfortunately, not garnered a lot of interest in the motor learning literature. Limited scientific work exploring the usefulness of the method has provided mixed reviews on its effectiveness over other therapeutic methods ${ }^{6,7}$. Nevertheless, Buchanan ${ }^{8}$, and Mattes $^{9}$, have recently made a stronger theoretical case for the method with recent empirical support ${ }^{10,11}$. These studies seem to argue in support of the idea that FM is deserving of greater scientific inquiry, which we hope to further add to here.

Given the wide scope of topics Feldenkrais spoke on as grounds/rationale for his method, only those most relevant for theories of action and perception will be discussed 
here. While arguably similar connections could be drawn to other theories on motor control, it is proposed here that the style of the "lessons" used in practice - exploration/search and the belief about how movements are controlled are integral to the foundational premise of SSA2.

In what follows, we will first present (non-exhaustively) the foundational premise of FM $8,12,13,14$. Second, we provide a review on the already proposed links to dynamical systems ${ }^{8,15}$, and show further similarities to $\mathrm{SSA}^{2}$. We also, then, point to some incongruencies between Feldenkrais' writings and SSA that should be highlighted if we are to be successful in making the link to SSA. Lastly, while FM already provides a means to demonstrate applicability of SSA, we describe viable ways in which FM might benefit from work done on SSA and vice-versa.

\section{THE FELDENKRAIS METHOD}

The Feldenkrais Method®, developed by Moshe Feldenkrais (1904 - 1984), is an approach to somatic education used in both performance ${ }^{9}$ and clinical ${ }^{8}$ settings to improve the efficiency of an individuals' movement 16,17 . "Efficient," or "ease," of movement in this case is reference to multiple factors such as muscular tonus, range-of-motion, stretch reflex, and force transmission. To Feldenkrais, the "feeling of muscular effort is not measuring work done, but something else. This 'something else' is how the movement is organized: it's quality"14(pg. 12). The foundational premise revolves around a notion of exploration and discovery of new movement possibilities by way of differentiating of perceptual information. Two different techniques are employed to achieve this purpose: Awareness Through Movement and Functional Integration.

\section{Awareness Through Movement}

Awareness Through Movement are group-based lessons where students are verbally guided through a series of movements aimed at promoting, firstly, an improved awareness of one's own movements via improved attunement to perceptual consequences of their movements and, second, discovery of new movement patterns. Feldenkrais' teachings often discuss the need to have no prior expectations about the form that any given movement should take - there is no "correct" movement. Clients are thus removed from preconceived notions about which muscle should be active during a given movement. Instead, they are offered the opportunity to discover which muscles are active during movements and, furthermore, they are invited to explore the relation of the resultant motions to the rest of the body and environment. A general example of this would be in a situation where students should have their feet some distance apart. In such a situation, it common for practitioners to instruct individuals to position their feet "hip-width" apart; in contrast, FM would encourage students to discover the width that is most comfortable to them ${ }^{14}$.

Movements performed in FM lessons provides students with kinesthetic information about their movements they were not previously attended to or were aware of - i.e. awareness through movement. Given that the focus of all FM work is to learn more efficient ways of doing, awareness that is achieved is often on perceiving tension in the muscles. For example, lying flat on the floor is used to provide proprioceptive information about distribution of points of contact with the ground, alluding to which muscles hold 
greater tension (cf. Brummer ${ }^{11}$ ). Becoming aware is not enough for change, however. Students must also learn what movement patterns, comparatively, are accomplished with less effort.

An example, adapted from discussion in Feldenkrais ${ }^{13(p g . ~ 89-119), ~ i l l u s t r a t e s ~ t h e ~}$ routine implemented to teach movements that progress from sitting to standing. Initially, students are to start seated on the floor, with knees bent, soles of the feet flat on the ground, and arms positioned behind the individual to support the torso upright. First, the movement starts by slowly tilting the knees to the left and right. This is done as many times as can be done without undue fatigue. Next, students continue these same movements of tilting the knees from side-to-side but allowing for more space between the knees so that they may both lay flat on either side. This will initiate a spiraling motion, lifting the pelvis forward.

At this point, attention (awareness) is brought to the support provided by the two arms; one is significantly more relied on to remain upright, while the other can be lifted away with no repercussions. Alternating side-to-side, provides information about how weight shifts with each tilting, and how tension is redistributed within the body. With knees to the left, the right arm is no longer needed for support; instead it is used to continue this spiraling motion to the left, by lifting it off the ground and swinging it in the direction of knees. From this position, the lesson is meant to teach a transition to standing. While there are no right or correct ways to stand from this position, how effortful the transition is will be a function of the chosen trajectory.

Eventually, as the spiral upward continues, the right hip will be high enough off the ground that the right foot can be lifted and moved in the same direction until it can be placed standing on the ground in front with the left foot soon to follow. With soles of both feet firmly on the ground, it becomes possible to stand up-right. At the completion of this movement you will have ended facing in the opposite direction. The whole movement can now be repeated many times, exploring variation in trajectory with each iteration.

Often, many more variants of this sequence, with additional sub-routines embedded in them, are used within the context of this sitting-to-standing lesson. This demonstrates the exploratory nature of Awareness Through Movement lessons, and how teachers guide awareness of students to different forms of perceptual information, but do not provide prescriptive instruction.

\section{Functional Integration}

Functional Integration is a complementary technique to Awareness Through Movement consisting of one-on-one sessions as opposed to group work. The Feldenkrais practitioner manually manipulate the student in order to gain information about the organization of the individual's musculoskeletal system. As is with the case of Awareness Through Movement, the goal in Functional Integration sessions is for the practitioner to establish efficient movement of the student. Those that are candidates for Functional Integration often have some form of chronic pain. The belief is that this chronic pain, being dependent on the habitual movement form, is so because the individual is unable to differentiate it from more efficient patterns. In other words, the movements may be so spastic or tonic that individuals are unable to perceive differences in movement quality as they are unable to differentiate what is intended movement from what is natural variation in the movement (see Withagen \& van Wermeskerken ${ }^{18}$ ). Functional Integration is meant to provide a learning environment that allows the individual to perceive these differences. 
With the system so constrained by undue stress, unable to reconcile the inefficiency itself, Functional Integration works on the premise that this stress needs to be alleviated before change can occur. How this is achieved is by supporting the individual in the least stressful position. If, for instance, one has complaints of lower back pain, having the feet elevated with knees and hips at 90 degrees is common to reduce discomfort. Providing support of this kind allows the musculoskeletal system to be more amenable to the practitioner's manipulations.

Functional Integration lessons utilize small amplitude slow motions as means for both the teacher and student to make better perceptual discriminations related to efficient movement. If practitioners feel hindered in their manipulations, and continued movement in the given direction would result in further resistance, then the involved limb is supported in such a way as to reduce tension until a trajectory of least resistance is discovered.

\section{PRELIMINARY COMPARISONS OF THE FELDENKRAIS METHOD AND DYNAMICAL SYSTEMS APPROACH}

\section{Habit as Attractors}

Dynamical systems approach views the human motor system in action as a dynamic system that is described by collective variables ${ }^{19,20}$. These capture the collective organization (coordination) of the system and allow characterization of how the system changes over time. Zanone and Kelso ${ }^{21}$ proposed that an account of learning should not be made on the assumption that a learner starts at a "blank slate" to be built up through experience, but instead learning occurs on a background of past experiences and previously learned coordination patterns. Initial tendencies (i.e., intrinsic dynamics ${ }^{19,20}$ ) are characterized by an attractor landscape that shows the stable modes of coordination one brings to the task ${ }^{22,23}$. The pathways of change with learning has been shown to be dependent on this initial composition of the behavioral repertoire22.

Buchanan and Ulrich ${ }^{15}$ and Reese ${ }^{24}$ have both likened the concept of attractors to what Feldenkrais discussed as habitual movement patterns. It is his belief that continuous use of given movement patterns leads to their eventual habituation, that is a preferred way of responding to environmental stimuli.

As the primary objective of FM is to develop an awareness of movement and discover new ways of acting, more often than not, when individuals that come to FM with some amount of pain or discomfort, it is believed that their current behavioral repertoire is "maladaptive." Feldenkrais ${ }^{12}$ suggested these maladaptive habits "can be likened to a groove into which the person sinks never to leave unless some special force makes him/her do so. With time, the groove deepens, and stronger forces are necessary to escape from it"12(pg. 118). In this way, "dysfunctional" movement patterns are thought to be a result of the repetitive use of an inappropriate movement pattern that has led to some impairment; in other words, it is a habit that must be "broken", or an attractor that must be destabilized.

\section{Awareness}

A core concept of FM is that change in movement coordination occurs as a result of enhanced awareness of how the movements are coordinated. Specifically, students are encouraged, during lessons, to explore and discover those movement patterns that are 
completed with least resistance or are the most efficient. Developing this perceptual awareness of our movement system is the goal of FM lessons.

What is the information gained from Awareness Through Movement lessons? Improved movement comes about from the enhanced detection of information contained in sensory motor stimuli. We contend that this information is of the same kind as that is discussed in ecological psychology25. From ecological psychology, what is perceived are perceptual invariants contained within patterned stimulus. In other words, there is no need for applying meaning to information-less stimuli at the receptors or development of a representation of the environment. Properties of the environment are perceived directly via structure contained within sensory stimuli 25,26 .

Examples of the type of awareness developed and information detected within Awareness Through Movement lessons similarly exemplify the relational quantities mentioned here. Considering the previous example of sitting-to-standing, the spiraling motion that ensues from the tilting of the knee; as attention is given to the changing points of contact with the ground and muscular tension distributed though out the body, information is provided on both motion relative to the environment and the self.

\section{SEARCH STRATEGIES APPROACH}

How an animal is able to interact with the environment (i.e. what actions are possible) is in part constrained by the environment. For instance, information contained within the ambient optic array ${ }^{25}$ specifies the layout of the environment and determine what actions are afforded for the particular individual. This gives rise to gradient and equilibrium regions of perception-action cycles available to the individual: the perceptual-motor workspace 27,28 . This workspace encompasses all possible movement forms that relate all perceptual and motor elements involved in a task, also characterizing the stability of those forms. For this reason, the perceptual-motor workspace can be seen as interchangeable to intrinsic dynamics of the individual22,27.

Newell et al. ${ }^{28(p g .101)}$ defined search strategies as "...the way in which an organism explores the perceptual-motor workspace of the organism-environment interaction to solve the motor problem." From Pacheco et al. ${ }^{2}$, it is also understood that the individual searches through the task space - the mapping that relates movement organization to task performance. Through the perception of the gradient and equilibrium regions of perceptualmotor and task spaces, individuals would perceive possibilities of change in their behavior to meet the demands of the task.

The term search refers to systematic changes within and between trials. SSA see search patterns as a window to the learning process. Identification of constraints that led to emergence of those patterns is, thus, tantamount. As one interacts with the environment in pursuit of a task goal, information variables that specify the success of the action are detected and influence whether change is required or not. Modifying the action will in turn provide new information and with time individuals will rely on more useful information for the task at hand.

\section{The Links Between SSA and FM}

Acquisition as search is an idea Feldenkrais would appreciate, and agree with: 
"Learning occurs when the nervous system repeats its exploratory activity on an object of the environment until it is successful, i.e. satisfies the intention. [...] There is thus a continuous interaction between the sensory and motor activities which are practically never independent" (Feldenkrais ${ }^{13}$, pg. 129).

The continuous interaction proposed between perception and action that leads to change - "satisfying the intention" - reflects a basic premise of SSA. It characterizes acquisition as a search process that leads to an increased coordination between perception and action ${ }^{29}$.

The link between SSA and FM is strengthened when one understands that both Awareness Through Movement and Functional Integration induce changes in how search is performed. By removing corrective feedback from the practice/learning environment, FM has, in the language of SSA, effectively reduced the constraints on search. In the case that movement forms are imposed on learners, the space searched is constrained as movement possibilities are eliminated by the instructional constraints. In modified version of what is usually called "discovery learning" 30 , instructions are provided throughout being guiding rather than corrective ${ }^{31}$. While, Feldenkrais practitioners do guide learners through the perceptual motor workspace, it would not be their intention for all learners to discover and exploit the same motor solution, as traditionally assumed in theories of motor learning 2 .

FM, in line to SSA, exploits the multiple solutions that can solve the same task (redundancy). The range of motor solutions available to a learner can be modified as a function of task constraints. Hristovski ${ }^{32}$ demonstrated how the probability of certain movement patterns changed as a function of distance to the target in a heavy bag boxing task. Evident from this work, is how modifying constraints (distance in the current example, and instructions in the case of Awareness Through Movement) can be used to expand or contract the space searched. The resultant from FM, thus, follows one of the arguments put forward in SSA: it is not necessary for persons learning the same skill to end up in the same region of the workspace.

Another feature that relates both SSA and FM is the fact that distinct initial conditions are not only expected but considered in how the practitioner expects changes to occur. Individuals will undoubtedly have started with different initial conditions and as a result certain information may not have been available to them. Take for example the lesson above that started by tilting the knees side to side. If you were to examine two different persons, one of whom had been a gymnast and the other was not an athlete, perform the same motion of tilting their knees, the gymnast may be able to drop both down to the floor while the other may only rotate several degrees. Certain kinesthetic information that comes from contact with the ground and larger amplitude motion is available only to the gymnast. This would not only result in differences in how they start performing but how movement patterns can/will change as practice develops.

Further links exist when considering the role of movement variability. FM sees early learning being facilitated by motor variability as it leads to the discovery of task relevant properties. From SSA, variability is seen as essential to perceive task relevant dimensions ${ }^{33}$. Feldenkrais discusses an "order-seeking function"13(pg. 131) exploring different motor solutions until the desired coordination is stabilized. Avoiding undesirable solutions is accomplished through variation along the order-seeking function (i.e. search). Several theoretical positions closely aligned with an ecological approach support the idea that variability supports an increased perception action coupling and avoid being stuck in local minima 34,35 . 


\section{INCONGRUENCIES BETWEEN FELDENKRAIS AND SSA}

While we believe his methods and practice closely resemble SSA, some areas of his work suggest that Feldenkrais was favorable to concepts that oppose in general dynamical systems. For instance, while the importance that Feldenkrais ${ }^{12,13,36}$ places on perceptual discriminations is readily apparent, there does not appear to be as direct a relation to dynamical systems and ecological approaches as Buchanan \& Ulrich ${ }^{8,15}$ claim. $^{2}$ Despite what is discussed on what awareness is brought about through movement, FM has a different view of what information, or lack thereof, is provided by environmental stimuli: "[...] at first, the stimulation of the senses carries no information other than the fact that the senses are being stimulated" 36 (pg. 47). This is at odds with theories of direct perception ${ }^{25}$ that state that through interaction with the environment individuals learn not to provide meaning to sensory stimulation (enriching), but to differentiate perceptual variables contained within the ambient array that supply information about properties of the environment.

Additionally, although Feldenkrais seems to consider coordination to be selforganized, in his rationalization for his method, Feldenkrais often discusses the storage of motor programs/engrams ${ }^{13}$. This is relevant as the ideas of self-organization in motor coordination and control explicitly do away with the notion of an executive or controlling agent, as well as localized stored solutions ${ }^{19}$.

Therefore, although we are inclined to link Feldenkrais' work to the SSA, one could just as easily approach the topic from an information processing perspective. Recent empirical examples testing FM predictions ${ }^{10,11}$ were not done from an ecological vantage point; thus, there is reason to consider FM as viable from multiple theoretical perspectives. Clearly, it is hard to envisage how motor programs would take advantage and explain how features such as exploration and variability favor rehabilitation in the ways described by Feldenkrais.

\section{FURTHER INTEGRATING FM AND SSA}

\section{From SSA to FM}

FM, as many theories in motor learning, emphasizes the possibility of varying conditions and, in some cases, argues for decomposition of a skill in its constituents (partwhole practice) to facilitate learning. As argued in $\mathrm{SSA}^{37}$, it is not that these tools are not fruitful, but they are dependent on the given task being practiced and learned. For variable practice, both FM and SSA must demonstrate how variable practice modifies the search process - not forgetting individual differences in the perceptual-motor workspace. For partwhole practice, FM must be able to say when such a procedure is appropriate for certain tasks and situations ${ }^{38}$. SSA can facilitate the process of understanding such procedures for theoretically grounded application. That is, FM researchers could implement the SSA in an applied situation and evaluate the search process employed when given manipulations occur. This would endow the practitioners with greater information about how to work with each client and lead to greater specialization of the method for each individual.

Beyond the actual manipulations, one must understand how transfer of practice is to occur. It is expected that through Awareness Through Movement or Functional 
Integration individuals would be able to modify movement patterns in contexts not directly practiced during the sessions. A great advance from SSA literature is the demonstration that performance on transfer tests is highly dependent on the search process implemented during practice ${ }^{39}$ and the solutions resultant of this search ${ }^{40}$. FM would be greatly supported if Awareness Through Movement and Functional Integration induce such search patterns related to improved transfer and/or understanding of what are the manipulations that would facilitate such patterns to occur.

\section{From FM to SSA}

An interesting topic that FM brings about and was never addressed within the SSA is the paired search process in Functional Integration. As the practitioner manipulates and moves the patient's limbs and joints, she/he acts according to what she/he perceives from the patient's response to those movements. Note that the patient is, as well, perceiving how the manipulations affect the current position of his/her limbs and how this is resisted given body current state and manipulation. The interaction between practitioner and patient would be characterized by a social perceptual-motor workspace on which movements, perception and change are shared between two individuals. A great challenge for SSA is to encompass such rich context. Additionally, it would be of great relevance to the area to understand how Functional Integration leads to topological changes in the perceptual-motor workspace. This would not only enrich SSA but would also provide a framework to work on social coordination dynamics beyond characterization of stable couplings (see above).

Additionally, in the same vein that transfer from SSA can help guide FM decisions in intervention, there are aspects of transfer that can be studied under SSA considering the positive results in FM. As described above, exercises promoted by FM are not practiced with the task on which improvements are to be observed. "Ease" or "efficiency" in movements, for instance, would be achieved in a large range of activities of daily living by intervening with a smaller set of exercises. Note that, although the exercises are driven in terms of a goal (e.g., sitting-to-standing), the result occurs in terms of ease of movement in broader terms and activities. SSA has not provided any insight yet of how search patterns in terms of task space allows improvement in aspects of movement not directly target by the task space (e.g., efficiency, variability, etc.).

\section{CONCLUDING REMARKS}

In this paper, we presented how FM and SSA are related by its theoretical premises and, doing this, demonstrated how SSA is/can be directly applied to practical settings. This has in no way been an admiration and blind support of FM as we have directed readers to both inconsistencies in theoretical foundation and incongruent empirical studies. We hope, instead, that this will garner scientific inquiry to both FM and SSA and, more importantly, will provide solid theoretical grounding for further discussion and application of the method. 


\section{REFERENCES}

1. Newell KM, McDonald PV, Kugler PN. The perceptual-motor workspace and the acquisition of skill. In: Requin J, Stelmach GE, eds. Tutorials in Motor Neuroscience. Netherlands: Kluwer Academic; 1991:95-108.

2. Pacheco MM, Lafe CW, Newell KM. Search strategies in the perceptual-motor workspace and the acquisition of coordination, control, and skill. Front Psychol. 2019;10:1874. doi:10.3389/fpsyg.2019.01874

3. Batson G, Deutsch JE. Effects of Feldenkrais awareness through movement on balance in adults with chronic neurological deficits following stroke: A preliminary study.

Complementary Health Practice Review. 2016;10(3):203-210. doi:10.1177/1533210105285516

4. Nair DG, Fuchs A, Burkart S, Steinberg FL, Kelso JAS. Assessing recovery in middle cerebral artery stroke using functional MRI. Brain Injury. 2005;19(13):1165-1176. doi:10.1080/02699050500149858

5. Stephens J, Davidson J, Derosa J, Kriz M, Saltzman N. Lengthening the hamstring muscles without stretching using "awareness through movement". Phys Ther. 2006;86(12):1641-1650. doi:10.2522/ptj.20040208

6. Ives JC, Shelley GA. The Feldenkrais Method(R) in rehabilitation: A review. Work. 1998;11(1):75-90. doi:10.3233/WOR-1998-11109

7. Ives JC. Comments on "the Feldenkrais Method: a dynamic approach to changing motor behavior". Res Q Exerc Sport. 2003;74(2):116-123 doi:10.1080/02701367.2003.10609072

8. Buchanan PA. The Feldenkrais Method of somatic education. In: A. B, ed. A Compendium of Essays on Alternative Therapy. InTech; 2012.

9. Mattes J. Attentional focus in motor learning, the Feldenkrais method, and mindful movement. Percept Motor Skill. 2016;123(1):258-276. doi:10.1177/0031512516661275

10. Verrel J, Almagor E, Schumann F, Lindenberger U, Kuhn S. Changes in neural resting state activity in primary and higher-order motor areas induced by a short sensorimotor intervention based on the Feldenkrais method. Front Hum Neurosci. 2015;9:232. doi:10.3389/fnhum.2015.00232

11. Brummer M, Walach $\mathrm{H}$, Schmidt $\mathrm{S}$. Feldenkrais 'functional integration' increases body contact surface in the supine position: A randomized-controlled experimental study. Front Psychol. 2018;9:2023. doi:10.3389/fpsyg.2018.02023

12. Feldenkrais M. Awareness Through Movement. New York, NY: HarperCollins; 1972.

13. Feldenkrais M. The Elusive Obvious. Capitola, CA: Meta Publications; 1981.

14. Feldenkrais M. Bodily expressions, 1988 (T. Hanna, Trans). In: Embodied Wisdom: The Collected Papers of Moshe Feldenkrais. North Atlantic Books; 2010:3-26.

15. Buchanan PA, Ulrich BD. The Feldenkrais Method: A dynamic approach to changing motor behavior. Res Q Exerc Sport. 2001;72(4):315-323. doi:0.1080/02701367.2001.10608968 
16. Hillier S, Worley A. The effectiveness of the Feldenkrais Method: A systematic review of the evidence. Evid Based Complement Alternat Med. 2015;2015:752160. doi: $10.1155 / 2015 / 752160$

17. Mohan V, Paungmali A, Sitilertpisan P, Henry LJ, Mohamad NB, Kharami NNB. Feldenkrais method on neck and low back pain to the type of exercises and outcome measurement tools: A systematic review. Polish Annals of Medicine. 2017;24(1):77-83. doi:10.1016/j.poamed.2016.10.003

18. Withagen $R$, van Wermeskerken $M$. Individual differences in learning to perceive length by dynamic touch: Evidence for variation in perceptual learning capacities. Atten Percept Psychophys. 2009;71(1):64-75. doi:10.3758/APP.71.1.64

19. Kelso JAS. Dynamic Patterns: The Self-Organization of Brain and Behavior. Cambridge, MA: MIT Press; 1995.

20. Thelen E, Smith LB. A Dynamic Systems Approach to the Development of Cognition and Action. Cambridge, MA: MIT Press; 1994.

21. Zanone PG, Kelso JAS. Evolution of behavioral attractors with learning: Nonequilibrium phase transitions. J Exp Psychol Hum Percept Perform. 1992;18(2):403-421. doi:10.1037/0096-1523.18.2.403

22. Kostrubiec V, Zanone PG, Fuchs A, Kelso JA. Beyond the blank slate: Routes to learning new coordination patterns depend on the intrinsic dynamics of the learner-experimental evidence and theoretical model. Front Hum Neurosci. 2012;6:222. doi:10.3389/fnhum.2012.00222

23. Zanone PG, Kelso JAS. Coordination dynamics of learning and transfer: Collective and component levels. J Exp Psychol Hum Percept Perform. 1997;23(5):1454-1480. doi:10.1037/0096-1523.23.5.1454

24. Reese M. The Feldenkrais Method and Dynamic System Principles. 1999. Available online at: http://davidzemach-bersin.com/wp-content/uploads/2012/02/Mark-Reese_FM-andDynamic-System.pdf [accessed 09/12/2019]

25. Gibson JJ. The Ecological Approach to Visual Perception. Hillsdale, NJ: Lawrence Erlbaum; 1979.

26. Michaels CF, Carello C. Direct Perception. Englewood Cliffs, NJ: Prentice-Hall; 1981.

27. Kugler PN, Turvey MT. Information, Natural Law, and the Self-Assembly of Rhythmic Movement. Hillsdale, NJ: Lawrence Erlbaum; 1987.

28. Newell KM, Kugler PN, van Emmerik REA, McDonald PV. Search strategies and the acquisition of coordination. In: Wallace SA, ed. Advances in psychology. Vol 61. NorthHolland 1989:85-122.

29. Shaw RE, Alley TR. How to draw learning curves: Their use and justification. In: Johnston T, Pietrewicz A, eds. Issues in the Ecological Study of Learning. Hillsdale, NJ: Lawrence Erlbaum; 1985.

30. Vereijken B, Whiting HTA. In defense of discovery learning. In: Wieringen PCW and Bootsma RJ ed. Catching Up: Selected Essays of HTA Whiting. 1989:155-169. 
Brazilian Journal of Motor Behavior

31. Kernodle MW, Carlton LG. Information feedback and the learning of multiple-degree-offreedom activities. J Motor Behav. 1992;24(2):187-195. doi: 10.1080/00222895.1992.9941614

32. Hristovski R, Davids K, Araujo D, Passos P. Constraints-induced emergence of functional novelty in complex neurobiological systems: A basis for creativity in sport. Nonlin Dynam Psychol. 2011;15(2):175.

33. Pacheco MM, Hsieh TY, Newell KM. Search strategies in practice: movement variability affords perception of task dynamics. Ecol Psychol. 2017;29(4):243-258. doi:10.1080/10407413.2017.1368354

34. Riccio GE. Information in movement variability about the qualitative dynamics of posture and orientation. In: Newell KM, Corcos DM, eds. Variability and Motor Control. Chicaco, IL: Human Kinetics; 1993.

35. Schöllhorn WI, Mayer-Kress G, Newell KM, Michelbrink M. Time scales of adaptive behavior and motor learning in the presence of stochastic perturbations. Hum Mov Sci. 2009;28(3):319-333. doi:10.1016/j.humov.2008.10.005

36. Feldenkrais M. On the primacy of hearing, 1976. In: Beringer E, ed. Embodied Wisdom. Berkley, CA: North Atlantic Books; 2010.

37. Pacheco MM, Newell KM. Learning a specific, individual and generalizable coordination function: evaluating the variability of practice hypothesis in motor learning. Exp Brain Res. 2018;236(12):3307-3318. doi:10.1007/s00221-018-5383-3

38. Kurtz S, Lee TD. Part and whole perceptual-motor practice of a polyrhythm. Neurosci Lett. 2003;338(3):205-208. doi:10.1016/s0304-3940(02)01394-0

39. Pacheco MM, Newell KM. Transfer as a function of exploration and stabilization in original practice. Hum Mov Sci. 2015;44:258-269. doi:10.1016/j.humov.2015.09.009

40. Pacheco MM, Newell KM. Transfer of a learned coordination function: Specific, individual and generalizable. Hum Mov Sci. 2018;59:66-80. doi:10.1016/j.humov.2018.03.019

Citation: Lafe CW, Pacheco MM. Applying the Search Strategies Approach to Practice: The Feldenkrais Method. BJMB. 2019: 13(5): 155-165.

Editors: Dr Fabio Augusto Barbieri - São Paulo State University (UNESP), Bauru, SP, Brazil; Dr José Angelo Barela São Paulo State University (UNESP), Rio Claro, SP, Brazil; Dr Natalia Madalena Rinaldi - Federal University of Espírito Santo (UFES), Vitória, ES, Brazil.

Copyright: @ 2019 Lafe and Pacheco and BJMB. This is an open-access article distributed under the terms of the Creative Commons Attribution-NonCommercial-NoDerivatives 4.0 International License which permits unrestricted use, distribution, and reproduction in any medium, provided the original author and source are credited.

Funding: MMP is supported by the Coordination for the Improvement of Higher Education Personnel [PNPD 2019].

Competing interests: The authors have declared that no competing interests exist.

DOI: https://doi.org/10.20338/bjmb.v13i5.147 